\begin{tabular}{c} 
International Journal of Advanced Mathematical Sciences, 6(1)(2018) 1-9 \\
International Journal of Advanced Mathematical Sciences \\
SPC \\
$\begin{array}{c}\text { Website: } w \text { ww.sciencepubco.com/index.php/IJAMS } \\
\text { doi: } 10.14419 / \text { ijams.v6il. } 8755 \\
\text { Research paper }\end{array}$ \\
\hline
\end{tabular}

\title{
Review on investigating the possibility of local hidden variable theories-quantum teleportation
}

\author{
Dorcas A. Addo ${ }^{1 *}$, Steve Abel ${ }^{2}$, Richard K. Ansah ${ }^{3}$ and Isaac Nkrumah ${ }^{4}$ \\ ${ }^{1}$ Department of Mathematics and Statistics, Kwame Nkrumah University of Science and Technology, Kumasi, Ghana \\ ${ }^{2}$ Department of Mathematics, Durham University, Durham \\ ${ }^{3}$ Department of Mathematics and Statistics, University of Energy and Natural Resources, Sunyani, Ghana \\ ${ }^{4}$ Department of Physics, Kwame Nkrumah University of Science and Technology, Kumasi, Ghana \\ *Corresponding author E-mail: dorcas@aims.edu.gh
}

\begin{abstract}
The core of the paper was to investigate the possibility of local hidden variable theory and its application in quantum teleportation. We reviewed literature on the Bell's inequality which is necessary for quantum teleportation. Quantum teleportation utilises a single-particle entangled state which can be successfully achieved by the application of the locality assumption which leads to Bell's inequality. A violation of the Bell's inequality signifies the nonlocal nature of a single particle useful for quantum teleportation.
\end{abstract}

Keywords: Bell's inequality; Classical Mechanics; Local Hidden Variable; Quantum Teleportation; Superpossition .

\section{Introduction}

The controversies surrounding the incompleteness of quantum mechanics and with the Einstein, Podolsky and Rosen (EPR) paradox led to several investigations, experiments and publications of findings in the EPR paradox and Bell's inequality and quantum teleportation. Home, D., and Selleri, F. (1991) [13] worked on Bell's theorem and the EPR paradox.

Blaylock (2010) [6] employed a simple and well-known thought experiment involving two correlated photons to help us focus on the logical assumptions needed to construct the EPR and Bell arguments. He presented in his paper that, the minimal assumptions behind Bell's inequality are locality and counterfactual definiteness, but not scientific realism, determinism, or hidden variables, as is often suggested. He also examined the resulting constraints on physical theory with an illustration from the many-worlds interpretation of quantum mechanics - an interpretation that we argue is deterministic, local, and realistic, but that nonetheless violates the Bell inequality.

Hasega et al., (2010) [12] measured the correlations between two degrees of freedom (comprising spatial and spin components) of single neutrons; which removes the need for a source of entangled neutron pairs. A Bell-like inequality was introduced to clarify the correlations that can arise between observables of otherwise independent degrees of freedom.

With quantum entanglement being a pure quantum phenomenon, many findings were discovered in the area of quantum information such us quantum computing, quantum cryptography and quantum teleportation. Quantum teleportation which is our subject of interest in this paper is a new area where more studies are made daily. Quantum teleportation uses shared entanglement between the sender (named Alice) and the remote receiver (Bob) as source.

After the work of Bennett et al. (1993) [5] teleportation of single-body quantum state via single quantum channel has received extent investigations both theoretically and experimentally. Since then, many scientist have worked in that area and new discoveries are been made every time.

Hu M. L. (2011) [15] studied standard quantum teleportation of one-qubit state for the situation in which the channel is subject to de-coherence, and a detailed calculation reveals that the quality of teleportation is determined by both the entanglement, and the purity of the channel state, and only the optimal matching of them ensures the highest fidelity of standard quantum teleportation.

The teleportation of an entangled two-body pure state by using two copies of Werner states as noisy quantum channels was considered by Lee and Kim, (2000) [18] and he then found out that, the quantum entanglement of the unknown state can be lost during the teleportation even when the channel is quantum correlated.

Horodeck et al., (1996) [14]. investigated the relations between teleportation, Bell's inequalities and inseparability and it was shown that for any mixed two spin- $\frac{1}{2}$ state which violates the Bell-CHSH inequality, then it is useful for teleportation. The result did also extended to any Bell's inequalities constructed of the expectation values of products of spin operators. 


\subsection{Objective}

Motivated by the works and findings above, the main objective of this work is to investigate the possibility of local hidden variable theory and its application will be in quantum teleportation. We will also explore some consequences of quantum mechanics and ways in which is it fundamentally different from classical mechanics, in particular due to superposition of states and the process of measurement. Then finally the study of Bell's inequality and the method by which a teleporting system can be created by the violation of Bell's inequality.

\section{Quantum mechanics vs. classical mechanics}

Classical mechanics solves problems in the macroscopic world. The Newtonian mechanics which had been firmly established as a valid theory for explaining the behaviours of all kinds of dynamic systems in the macroscopic world, could not give a valid explanation for experimental anomalies. Explaining these anomalies led to the rise of quantum mechanics (Reilly, 1970) [20]. Quantum mechanics requires an ordinary prescription for operators and none ordering prescription respects the symmetries of the classical theory (Terhal et al., 2003) [25].

Quantum mechanics deals with physical phenomena at nanoscopic scales:- in the order of Planck constant $(h)$ and it gives a mathematical description of particle like and wavelike behaviour and interactions of matter and energy.

Quantum mechanics has gone beyond the illusion of Newton's universe. It has revealed that

(i) atomic matter, dissolves into waves of potential existence.

(ii) determinism falls apart, giving us a spontaneous world.

(iii) we are living a world in which the observed phenomena depends on how we choose to look at things.

(iv) quantum mechanics is a world of "possibility". Eg. quantum tunnelling:- the phenomenon that real - energy classical particles can never enter classically forbidden regions.

"The Newtonian laws that govern the universe everyday don't hold true on the quantum level".

Ways in which quantum mechanics is different from classical mechanics will be explained from the angle of

- Superposition of states and

- Process of measurement.

\subsection{Superposition of states}

One of the basic principles of quantum mechanics is the superposition principle. It is the relation that exist among states.

Example is the quantum qubit (the corresponding unit of quantum information) state which is a linear superposition of the basis state represented as a linear combinations of $|0\rangle$ and $|1\rangle$. Thus mostly expressed in the normalized state as

$|\Psi\rangle=\alpha|0\rangle+\beta|1\rangle$,

where $\alpha$ and $\beta$ represent the probability of amplitudes in the complex form satisfying $|\alpha|^{2}+|\beta|^{2}=1$.

Qubits represent atoms, ions, electrons or photons in their respective control devices that are working to act as a computer memory. Measuring the state (1) results in $|0\rangle$ with probability $|\alpha|^{2}$, and $|1\rangle$ with probability $|\beta|^{2}$. Except in cases where $\alpha=0$ and $\beta=0$, that the measurement constantly disturbs the state. After measurement, the system is in the measured state.

A classical bit differs from a qubit; a classical bit can be measured without disturbing it and we can decrypt all of the information that it encodes. We can completely encode one bit in one qubit. A qubit can hold more information, for example up to two bits using super-dense coding (a technique used to send two bits of classical information using one qubit). Whereas the state of a bit is either 0 or 1 , the state of a qubit can be a superposition of both. Supposing the value of a classical bit is initially unknown to us, then based on the information available to us, we can say there is a probability $p_{0}$ that has the value 0 , and a probability $p_{1}$, that the bit has the value 1 , where $p_{0}+p_{1}=1$.

\subsection{Implications of superposition}

In superposition principle, quantum mechanics is fundamentally different from classical mechanics. Newton's second law $(F=m a)$ which states that the acceleration produced by a net force is directly proportional to the magnitude of the net force in the same direction as in the net force is the equation of motion used mathematically in classical mechanics. It predicts what the system will do at any time after the initial condition.

The Schrödinger equation for quantum system is the analogue of Newton's law. The Schrödinger equation describes how wave propagates and behaves, the wave is described by the wave function and the equation governing it is called the Schrödinger wave equation. It gives a consistent explanation with both classical mechanics and special relativity.

The general time dependent Schrödinger equation (TDSE) is expressed as

$i \hbar \frac{\partial}{\partial t} \Psi=\hat{H} \Psi$

and the non relativistic equation is expressed in the form

$i \hbar \frac{\partial}{\partial t} \Psi(r, t)=\left[-\frac{\hbar^{2}}{2 \mu} \nabla^{2}+V(r, t)\right] \Psi(r, t)$.

The time independent Schrödinger equation (TISE) in the general form is

$E \Psi=\hat{H} \Psi$

and its non relativistic equation is in the form

$E \Psi(r)=\left[-\frac{\hbar^{2}}{2 \mu} \nabla^{2}+V(r)\right] \Psi(r)$.

Quantum mechanics incorporates four classes of phenomena for which classical mechanics can not account for:

- Quantization: It predicts only specific discrete values can occur when certain properties of the system are measured. It is a phenomenon used in quantum mechanics. Examples are energy quantization $(E=\hbar w)$ and quantization of angular momentum.

- Measurement and Uncertainty: Quantum mechanics talks about probability as a particle property can not be determined exactly (that is, you can not measure position and momentum at the same time) as compared to classical mechanics (the particle has an exact position and momentum using Newton's laws). The uncertainty equation is expressed as

$\triangle P_{x} \triangle x \geq \frac{\hbar}{2 \pi}$ 
- Quantum Tunneling: It describes a phenomenon where a particle can penetrate through a potential barrier. It does not have a classical counterpart since a ball roll back to its initial position because it does not have enough energy to get over the hill to the other side. It is possible in quantum mechanics what seems impossible in the classical world.

- Particle as wave: It is the property that a single element has both particle-like and wavelike properties. It explains classical mechanics inability of "wave" and "particle" completely describing the nature and behaviour of nanoscopic (quantum) objects. As written by Einstein "It seems as though we must use the one theory and sometimes the other, while at times we may use either. We are faced with a new kind of difficulty. We have two contradictory pictures of reality; separately neither of them fully explains the phenomenon of light, but together they do".

\subsection{Process of measurement}

The representation of the states and the measurement process in quantum mechanics is very counterintuitive. This is mainly due to the difference between the treatments of classical and quantum mechanics.

\subsection{Measurements in classical mechanics}

Properties such as position, momentum and energy can be measured in any degree of accuracy desired (Griffiths, 2005) [5]. In classical measurement, there is interference or pre-existing account of the behaviour of the measurement property and it is often considered deterministic, real and local which that of the quantum measurement process is also considered as random and indeterministic. The predicted values of the measurement in quantum mechanics are described by a probability distribution (Wakita, 1960) [26]. Observables are represented as scalar quantities of classical mechanics as opposed to self adjoint operators of quantum mechanics.

A pair of numbers $(x, p)$ is used to represent a state of a system in classical mechanics. The pair of numbers contains every necessary information needed about that system in one dimension.

\subsection{Representation of state and observables}

"Measurement causes the system to jump to eigenstate of the dynamic variable that is been measured, the eigenvalue this eigenstate belongs to being equal to the result of the measurement", (Dirac, 1981) [8]. Measurement in quantum mechanics are values which are stated as postulates.

A wave-function $\Psi(x)$ is used in quantum mechanics instead of two real variables. The wave-function $\Psi(x)$ is the state of quantum mechanics that gives all information about that particle.

Classical Mechanics: state $\Leftrightarrow(x, p)$

Quantum Mechanics: state $\Leftrightarrow \Psi=\Psi(x)$

Observable properties in classical mechanics is represented by a function of the state, that is position and momentum $(x, p)$. Example is the energy of a given particle by the Hamilton function

$E=H(p, x)=\frac{p^{2}}{2 m}+V(x)=\frac{m v^{2}}{2}+V(x)$.

Observables are represented by linear operators in quantum mechanics.

$\hat{A}\left(c_{1} \Psi_{1}+c_{2} \Psi_{2}\right)=\hat{A} c_{1} \Psi_{1}+\hat{A} c_{2} \Psi_{2}$

$\hat{A}(\mathrm{~cm})=c \hat{A} m$
The equation 3 is satisfied for all complex numbers $c_{1}, c_{2}$ and "functions" $\Psi_{1}$, and $\Psi_{2}$. Quantum mechanics is a linear theory. The first postulates states that,

"for a physical system, any possible state of the system is represented by a wave function $\Psi "$ (S. Turgut, 2013) [23].

$\Psi$ assumes different mathematical structures depending on how the system is described. $\Psi$ might represent a column matrix where all its entries are either numbers or functions.

Considering a single particle, $\Psi$ is normally represented as a function of position $\Psi(x)$ in one dimension and in three dimension as $\Psi(x, y, z)$. For two particles, $\Psi$ is then a function of both positions (i.e., $\left.\Psi=\Psi\left(x_{n}, y_{n}, z_{n}\right)\right)$ denoting the position of the $n$-th particle.

Both the classical and quantum mechanical states $\Psi$ depends on time. A particle in one dimension for a quantum state is represented as $\Psi=\Psi(x, t)$. The classical state in one dimension $(1 D)$ is $(x, v)$. As the particle move, $x$ changes and therefore, the state is time dependent.

For any observable property $A$ of a system, there is a corresponding operator $\hat{A}$.

Typical observables are position, momentum and energy. Considering $\hat{A} \Psi=\phi$, if $\Psi$ is a function of position $x$, i.e., $\Psi=\Psi(x)$, then so is $\phi$. The operator $\hat{A}$ acts on the wave-function like mathematical objects and produces a mathematical object of the same type. $\Psi$ can also be considered as an elements of vector space. We can form linear combinations of of $\Psi_{1}$ and $\Psi_{2}$ to obtain another possible wave-function $\Psi=c_{1} \Psi_{1}+c_{2} \Psi_{2}$. Thus the wave function can also be called vectors. The term given for this linear combination is usually called superposition. In $\hat{A} \Psi=\phi$, the operator acts on a function and produces another function.

In quantum mechanics, the state of a system on the atomic and subatomic scale is not defined by a set of dynamic variables each with a specific numerical value specified by "state function", (Tang, 2005) [24].

\subsection{States where observables are definite}

If the equation $\hat{A} \phi=\lambda \phi$ is satisfied for a complex number and nonzero function $\phi$, then $\lambda$ is the eigenvalue of the observable $\hat{A}$ and $\phi$ is the eigenvector. The set of all eigenvalues $\lambda$ forms the spectrum of $\hat{A}$.

If we consider $\Psi$ to be an eigenvector of an observable $\hat{A}$ with eigenvalue $a$, then the equation

$\hat{A} \Psi=a \Psi$

is satisfied. Thus the observable $\hat{A}$ has the definite value $a$ in the state $\Psi$. The system in the state $\Psi$ when an observable $\hat{A}$ is measured implies that

- getting the value $a$ with certainty and

- the system remains in the same state.

Example: Considering a particle in one dimension, the momentum operator $\hat{p}_{x}=\hat{p}$ is expressed as

$\hat{p}=\frac{\hbar}{i} \frac{\partial}{\partial x}$.

The eigenvalue is usually expressed as $\hbar k$ where $k$ is the wave number. The corresponding eigenfunction as $\phi_{k}$ is given as

$$
\begin{aligned}
\hat{p} \phi_{k} & =\hbar k \phi_{k}, \\
\phi_{k}(x) & =A e^{i k x} .
\end{aligned}
$$

The function above is the Broglie relation having a single wavelength $\lambda=\frac{2 \pi}{k}$. 
If we consider a particle in the state $\Psi=4 e^{i k x}$, then the momentum has a definite value of $\hbar k$ and the constant 4 has no effect on the statement because it does not change the fact that $\Psi$ is an eigenvector of $\hat{p}$.

If $\phi$ is an eigenvector of $\hat{A}$ with eigenvalue $\lambda$, then for any non-zero complex number $c$ the function $c \phi$ is also an eigenvector $\hat{A}$ with the same eigenvalue $\lambda$.

$\hat{A} \phi=\lambda \quad \Rightarrow \quad \hat{A}(c \phi)=\lambda(c \phi)$

\section{EPR experiment, Bell's theorem and Bell's inequality}

The question is whether quantum mechanics is an incomplete theory, whether it is non-local and whether it can be explained realistically. The EPR experiment seeks to answer the bewildering interpretations given by physicist and philosophers as to the possibility of a local hidden variable and incompleteness.

"Theoretical physicist live in a classical world, looking out into a quantum mechanical world. Nobody knows just where the boundary between classical and quantum mechanics is situated. Most feel the experiment switch setting and pointer readings are on this side. But some would think the boundary nearer, others would think it is farther, and many would prefer not to think about it", (Bell, 2004) [3]

"To know the quantum mechanical system of a state implies, in general, only statistical restrictions on the results of measurement, it seems interesting to ask if this statistical element be thought of as arising, as in classical mechanics, because the states in question are averages over better states for which individually the results would be quite determined. These hypothetical 'dispersion free' states would be specified not only by quantum mechanical state vector but also by additional 'hidden variables'- hidden because if states with prescribed values of these variables could actually be prepared, quantum mechanics would be observable inadequate (Bell, 1982) [4]."

The two quotes above are from the introduction of John Bell's collection of papers. It addresses the so-called incompleteness of whether quantum mechanics is a complete physical theory.

Definition 3.1. (Incompleteness problem, preliminary formalism). "Is quantum mechanics a complete physical theory?"

What do we mean when we talk of a complete physical theory? Does quantum mechanics really give a comprehensive description of the physical phenomena? The ambiguity of this formulation is only preliminary.

Following John Bell's (2004) [3] paper, the motivation to study hidden local variable - was an attempt to the incompleteness problem - and the motivation was distinguished into three categories.

1. The likelihood of a unitary account of the world and the desire to dichotomize the physical world into classical and quantum mechanics. Bell put it as: "it is the possibility, of a homogeneous account of this world, which is for me the chief motivation of the study of the so-called 'hidden-variable' possibility, (Bell, 2004) [3]". The possibility that the world exist as a uniform or single entity and things are correlated.

2 . To disencumber the statistical element of quantum mechanics and get some form of realism and or determinism back into the realm of micro-physics. That the behaviour of physical objects could be principally ascertain by physical laws.

3 . To come to terms with some uncanny quantum mechanics predictions that deal with the notion of non-locality, "we seem almost to cry out for a hidden local variable interpretation (Bell, 2004) [3]" in Bell's own words. It had to do with some quantum mechanical relations exhibiting non-locality that the EPR addresses in their paper (Einstein et al., 1935) [9]. Nonetheless quantum mechanics does not specify these variable and one is left to doubt even non-local correlations. EPR argues that, following the principle of scientific realism, that quantum mechanical theory fails to specify an element of reality. Thereby implying, as a physical theory, it is incomplete. An example is seen in the orthodox interpretation of quantum mechanics that claims that the polarizations of two photons are not well specified before measuring it. The entangled superposition of $|x\rangle$ and $|y\rangle$ of the two photons. Both photons are said to collapse only when one measurement takes place. Quantum mechanics does not define what the polarizations are and does not even accept the definite polarization of either photon.

Bell's motivation to study hidden local variable formalism, fix the quantum formalism of the then called local realism.

Definition 3.2. (Local realism:) All correlations in correlation tables for all physical systems obtained from actual or possible physical measurements allow a local hidden variable (HV) - model.

\section{$P_{A, B}(a, b \mid \lambda)=P_{A}(a \mid \lambda) P_{B}(b \mid \lambda)$}

The outcomes of $a$ and $b$ for a fixed $\lambda$ are completely statistically independent which is referred to as factorisability. This means that the hidden variable for quantum mechanics seeks for a local realistic account of the quantum phenomena. It gave room to the question, "can one really answer the incompleteness problem with local hidden variables?". Thus three great scientists, Einstein, Podolsky and Rosen, (1935) [9] were the first to say quantum mechanics can not be a complete theory but should be added up by additional variable.

\subsection{EPR experiment}

The EPR experiment was formulated in 1935 and their argument was that "Quantum mechanics cannot be a complete theory of the physical world". It drew attention to Quantum Entanglement (which quantum mechanics predict) in those days. Their argument was based on the analysis of measuring the property of a particle (two separate particles but correlated) which interacted in the past and then separated. When one particle is measured, it will have effect on the other particle (spooky-action-at-a-distance) which Einstein could not accept due to the "principle of locality" which states that, action at one location does not have any effect at a separate location.

Thus all particles possessed some sort of fixed properties which were hidden from our view (hidden variable).

Bohm in 1950 reformulated the EPR experiment as measurements of spin $\frac{1}{2}$ particles instead of the continuous range of results using colour experiment.

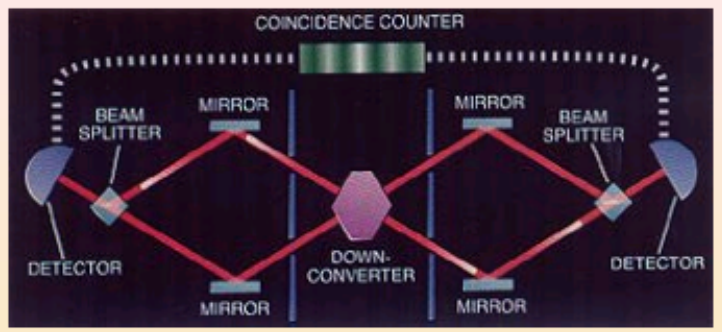

Figure 1: Bohm EPR colour experiment (Einstein et al., 1935) [9] 
If a single particle splits into two: one red and one green, and red and green are replaced by spin up and spin down respectively showing their state of measurement and a white particle is a combination of these two colours in figure 1. If the two particles move in opposite direction and we do not know which colour is which, then one particle is measured to be spin up, then the other spin down. Using Heisenberg's uncertainty principle in quantum mechanics, it could be either spin up or spin down depending on the time of measurement. Through the hidden variable parameter, one particle had always been spin up in one direction and the other spin down in the opposite direction. The laws violated by quantum mechanics in the EPR experiment is the principle of locality and Heisenberg uncertainty principle.

The EPR experiment defines determinism:- that is complete knowledge of the current state of a physical system is sufficient to determine the future state of the system. And if one can also determine certain parameters without interfering with the system those parameters must be 'real'. Considering a theory to be complete implies that, it should be able to predict all real parameters, including the polarizations in Bohm's EPR experiment. But QM does not predict the polarizations hence considered to be incomplete. This assumption, when combined with the conclusions of special relativity, implies that no effect can travel faster than the speed of light.

For a complete theory, "every element of the physical reality must have a counterpart in the physical theory".

EPR is not a no-go theorem for hidden variable models of quantum mechanics but it argues that there must be a more complete description of physical reality behind quantum mechanics.

\subsection{Bell's theorem}

Definition 3.3. Bell theorem states that no physical theory of local hidden variables can ever reproduce all the predictions of quantum mechanics (Bell, 1964).

When the EPR paper was first published, Bell was concerned if the whole EPR experiment can be accommodated in a world which is compatible with the world-view (classical world) of pre-quantum mechanics physics. The classical world is local, deterministic and Markovian (LDM) which satisfies the assumption that: Our world is

1 . Local:- No direct casual connection between spatially separated events (Genovese, 2005) [10].

2 . Deterministic:- knowledge of a current state of a physical system to determine the future state of the system.

3 . Markovian:- All necessary information from the past is encoded in the state of affairs in the present.

Bell considered polarization measurements for twin state photons at arbitrary angles $\theta_{1}, \theta_{2}$. The twin state is represented by an entangled isotropic state in quantum mechanics as

$\Psi=\frac{1}{\sqrt{2}}\left(|x\rangle_{1}|x\rangle_{2}+|y\rangle_{1}|y\rangle_{2}\right)$,

where $|x\rangle_{i}$ is the polarization state of the ith photon through a horizontal filter and absorbed by a vertical filter and $|y\rangle_{i}$ represents a polarization state of the ith photon through a vertical filter and absorbed by a horizontal filter. Quantum mechanics' predictions about the correlations of polarization are different from correlated predictions by common sense. Considering the measurement of twin state photons with polarizing filters oriented at different angles $\theta_{1}$ for the first photon and $\theta_{2}$ for the second photon. Which ever measurement is performed first collapses the entangled twin state superposition to a single polarization state which is identical to both
Table 4: $\theta_{1}=-\alpha, \theta_{2}=\alpha$ avg. mismatch $\leq 50 \%$

\begin{tabular}{|l|l|l|l|l|l|l|l|l|l|l|l|l|l|l|l|l|l|l|l|l|l|l|l|l|}
\hline Filter 1 & 0 & $\mathbf{0}$ & $\mathbf{0}$ & 0 & 0 & 1 & 0 & 1 & $\mathbf{1}$ & 1 & 1 & 1 & 0 & 1 & 0 & $\mathbf{1}$ & $\mathbf{1}$ & 0 & 1 & 0 & 1 & $\mathbf{0}$ & $\mathbf{1}$ & 1 \\
\hline
\end{tabular}

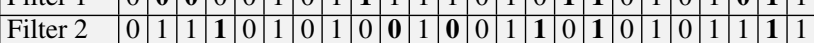

according to quantum mechanics. If the wave is polarized along $\theta_{2}$ direction, the amplitude along $\theta_{1}$ is $\cos \left(\theta_{2}-\theta_{1}\right)$. Quantum mechanics predict that the probability for transmission given by a wave amplitude squared is $\operatorname{Prob}\left(M_{1}(\theta)=M_{2}(\theta)\right)=\cos ^{2}\left(\theta_{2}-\theta_{1}\right)$.

What does common sense predict? Common sense predict that,

$\operatorname{Prob}\left(M_{1}(\theta)=M_{2}(\theta)\right)$ is $100 \%$ coincidence.

The measurement with filters along the same direction gives the probability of obtaining the same measurement.

Table 1: Sequence of both being absorbed (0) or both transmitted (1)

\begin{tabular}{|l|l|l|l|l|l|l|l|l|l|l|l|l|l|l|l|l|l|l|l|l|l|l|}
\hline Filter 1 & 0 & 1 & 1 & 0 & 0 & 1 & 0 & 1 & 0 & 1 & 1 & 1 & 0 & 1 & 0 & 0 & 0 & 0 & 1 & 0 & 1 & 1 \\
\hline
\end{tabular}

\begin{tabular}{|l|l|l|l|l|l|l|l|lllllllllllllllll}
\hline Filter 2 & 0 & 1 & 1 & 0 & 0 & 1 & 0 & 1 & 0 & 1 & 1 & 1 & 0 & 1 & 0 & 0 & 0 & 0 & 1 & 0 & 1 & 1 & 0 & 1 \\
\hline
\end{tabular}

We also know that paired measurements with perpendicular orientation of the filters will always give an opposite results, that is when one photon is absorbed, the other is transmitted.

Let's say the filters differ in angles, that is for $\theta_{2}-\theta_{1}$ between $0^{\circ}$ and $90^{\circ}$ will yield an intermediate correlations.

$\operatorname{Prob}\left(M_{1}(\theta)=M_{2}\left(\theta+90^{\circ}\right)\right)$ is $100 \%$ coincidence. The coincidence is between $0 \%$ and $100 \%$.

For example, let $\alpha$ be some angle for which the mismatch is $25 \%$ (averagely) and the coincidence is $75 \%$. The sequence for filter 1 oriented at $\theta_{1}=0$ and filter 2 oriented at an angle $\theta_{2}=\alpha$ might also look like Table 2

Table 2: $\theta_{1}=0, \theta_{2}=\alpha$ avg. mismatch $25 \%$

\begin{tabular}{|l|l|l|l|l|l|l|l|l|l|l|l|l|l|l|l|l|l|l|l|l|l|l|l|l|}
\hline Filter 1 & 0 & 1 & 1 & 0 & 0 & 1 & 0 & 1 & 0 & 1 & 1 & 1 & 0 & 1 & 0 & 0 & 0 & 0 & 1 & 0 & 1 & 1 & 0 & 1 \\
\hline
\end{tabular}

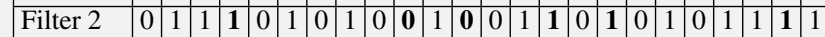

where the boldface entries highlight where there is disagreement between the two sequences in table 2 .

Table 3: $\theta_{1}=-\alpha, \theta_{2}=0$ avg. mismatch $25 \%$

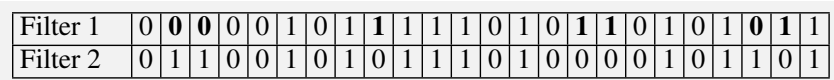

We expect that the measurement disagreement should be $50 \%$ averagely. This means that the mismatch between measurements with $\theta_{1}=-\alpha$ and $\theta_{2}=\alpha$ should be less or equal to $50 \%$ in table 3 This is an example of Bell's inequality.

The "common sense" predict that the mismatch should be at most $50 \%$ which quantum mechanics (experimental) predicts a mismatch of $75 \%$ in table 4 .

For $\alpha=30^{\circ}$, coincidence is $75 \%$ and mismatch is $25 \%$.

Coincidence rate predicted by quantum mechanics is $\cos ^{2}\left(\theta_{2}-\theta_{1}\right)$ where $\theta_{1}=\alpha$ and $\theta_{2}=0$ or vice versa

$\Rightarrow \quad \cos ^{2}\left(30^{\circ}\right)=0.75$. 
For $\theta_{1}=-30^{\circ}$ and $\theta_{2}=30^{\circ}$,

$\Rightarrow \cos ^{2}\left(30^{\circ}+30^{\circ}\right)=\cos ^{2}\left(60^{\circ}\right)=25 \%$. The mismatch rate is $75 \%$ which is not less than $50 \%$ as predicted by common sense.

\subsection{Bell's inequality}

This section is based on the original paper of Bell (Bell et al., 1964). A linear polarization measurement on the two photons as observed in figure 2, with filters 1 and 2. The filter 1 , in orientation $\mathbf{a}$, is followed by two detectors, given the result 1 or 0 which corresponds to a linear polarization parallel or perpendicular to $\mathbf{a}$. The second filter in orientation b also acts similarly (Alain, 2002) [1]. The Bell's inequality requires absence of communication between measurement sites.

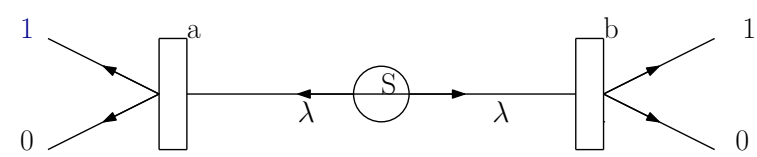

Figure 2: Einstein-Podolsky-Rosen-Bohm Gedanken experiment with photons (Einstein et al., 1935)

$A(\vec{a}, \lambda)=1,0$ at polarizer 1

$B(\vec{b}, \lambda)=1,0$ at polarizer 2

Transmission when $a=b \quad \Rightarrow \quad B(\vec{b}, \lambda)=A(\vec{a}, \lambda)$

If $\rho(\lambda)$ is the probability distribution of $\lambda$ (the hidden variable) then the expectation value of the product of the two components is

$\Pi(\vec{a}, \vec{b})=\int d \lambda \rho(\lambda) A(\vec{a}, \lambda) B(\vec{b}, \lambda)$.

So, the result depends on the angle between $\lambda$ and polarizer orientation.

Equation (5) should be equal to the quantum mechanical expectation value, which for a single state is

$\left\langle\vec{\sigma}_{1} \cdot \vec{a} \vec{\sigma}_{2} \cdot \vec{b}\right\rangle=-\vec{a} \cdot \vec{b}$

where $\sigma_{1}$ and $\sigma_{2}$ are selected components of the spins.

Quantum mechanics predicts

$\Pi(\vec{a}, \vec{b})=\cos ^{2} \theta_{a b}$

But no hidden local variable $(\lambda)$ can reproduce quantum mechanics predictions at all orientations as in (3.2).

If $\rho(\lambda)$ is normalized, the probability distribution is,

$\int d \lambda \rho(\lambda)=1$

and because of the equation (4) are valid, (5) cannot be less than -1 . It could reach the value -1 only for $\vec{a}=\vec{b}$ if

$A(\vec{a}, \lambda)=-B(\vec{b}, \lambda)$,

except for a set of points $\lambda$ of zero probability.

Thus (5) becomes

$\Pi(\vec{a}, \vec{b})=-\int d \lambda \rho(\lambda) A(\vec{a}, \lambda) B(\vec{b}, \lambda)$.

If we define $\vec{c}$, another unit vector; and with the help of (4),

$\Pi(\vec{a}, \vec{b})-\Pi(\vec{a}, \vec{c})=-\int d \lambda \rho(\lambda)[A(\vec{a}, \lambda) A(\vec{b}, \lambda)-A(\vec{a}, \lambda) A(\vec{c}, \lambda)]$

$=\int d \lambda \rho(\lambda) A(\vec{a}, \lambda) A(\vec{b}, \lambda)[A(\vec{b}, \lambda) A(\vec{c}, \lambda)-1]$, from which

$|\Pi(\vec{a}, \vec{b})-\Pi(\vec{a}, \vec{c})| \leq \int d \lambda \rho(\lambda)[1-A(\vec{b}, \lambda) A(\vec{c}, \lambda)]$.

For (3), the second term of the right hand side of the equation (7) is equal to $\Pi(\vec{b}, \vec{c})$, then

$1+\Pi(\vec{b}, \vec{c}) \geq|\Pi(\vec{a}, \vec{b})-\Pi(\vec{a}, \vec{c})|$.

The inequality (8) is the Bell's inequality.

Violation by Quantum Mechanics: Let the unit vectors $\vec{a}, \vec{b}, \vec{c}$ lie into right circular cones with small width - since the directions are physically affected by certain errors. Considering the averages of (5) and (6) we have

$\bar{\Pi}(\vec{a}, \vec{b}), \quad-\overrightarrow{\vec{a} \cdot \vec{b}}$,

where the bar denotes the independent averages of $\Pi\left(\vec{a}^{\prime}, \vec{b}^{\prime}\right)$ and $-\vec{a}^{\prime} \cdot \vec{b}^{\prime}$ over vectors $\vec{a}^{\prime}$ and $\vec{b}^{\prime}$ within specified angles of $\vec{a}$ and $\vec{b}$. For any unit vector $\vec{a}$ and $\vec{b}, \Pi(\vec{a}, \vec{b})$ approximates quantum mechanical results well. Thus the average is bounded on the upper side by a small quantity $\varepsilon$ :

$|\bar{\Pi}(\vec{a}, \vec{b})+\overline{\vec{a} \cdot \vec{b}}| \leq \varepsilon$.

We want to show that $\varepsilon$ cannot be made small, therefore the impossibility of (5) to be equal to (6).

Consider

$|\overrightarrow{\vec{a} \cdot \vec{b}}-\vec{a} \cdot \vec{b}| \leq \delta$

then

$|\bar{\Pi}(\vec{a}, \vec{b})+\vec{a} \cdot \vec{b}| \leq|\bar{\Pi}(\vec{a}, \vec{b})+\overrightarrow{\vec{a} \cdot \vec{b}}|+|\vec{a} \cdot \vec{b}-\overrightarrow{\vec{a} \cdot \vec{b}}| \leq \varepsilon+\delta$.

From (6), we have

$\bar{\Pi}(\vec{a}, \vec{b})=\int d \lambda \rho(\lambda) \bar{A}(\vec{a}, \lambda) \bar{B}(\vec{b}, \lambda) \leq 1$,

where

$|\bar{A}(\vec{a}, \lambda) \leq 1|, \quad|\bar{B}(\vec{b}, \lambda) \leq 1|$.

From (11) and (12), let $\vec{a}=\vec{b}$, we have

$\int d \lambda \rho(\lambda)[\bar{A}(\vec{b}, \lambda) \bar{B}(\vec{b}, \lambda)+1] \leq \varepsilon+\delta$.

From equation (12) we have

$\bar{\Pi}(\vec{a}, \vec{b})-\bar{\Pi}(\vec{a}, \vec{c})=\int d \lambda \rho(\lambda)[\bar{A}(\vec{a}, \lambda) \bar{B}(\vec{b}, \lambda)-\bar{A}(\vec{a}, \lambda) \bar{B}(\vec{c}, \lambda)]$

$=\int d \lambda \rho(\lambda) \bar{A}(\vec{a}, \lambda) \bar{B}(\vec{b}, \lambda)[1+\bar{A}(\vec{b}, \lambda) \bar{B}(\vec{c}, \lambda)]$

$-\int d \lambda \rho(\lambda) \bar{A}(\vec{a}, \lambda) \bar{B}(\vec{c}, \lambda)[1+\vec{A}(\vec{b}, \lambda) \bar{B}(\vec{b}, \lambda)]$.

Considering (13), we arrive at

$|\bar{\Pi}(\vec{a}, \vec{b})-\bar{\Pi}(\vec{a}, \vec{c})| \leq \int d \lambda \rho(\lambda)[\bar{A}(\vec{b}, \lambda) \bar{B}(\vec{c}, \lambda)]$

$+\int d \lambda \rho(\lambda)[1+\bar{A}(\vec{b}, \lambda) \bar{B}(\vec{b}, \lambda)]$

Using (12) and (14),

$|\bar{\Pi}(\vec{a}, \vec{b})-\bar{\Pi}(\vec{a}, \vec{c})| \leq 1+\bar{\Pi}(\vec{b}, \vec{c})+\varepsilon+\delta$.

From (11), the right hand side will be,

$1+\bar{\Pi}(\vec{b}, \vec{c})+\varepsilon+\delta=1+\bar{\Pi}(\vec{b}, \vec{c})+\vec{b} \cdot \vec{c}-\vec{b} \cdot \vec{c}+\varepsilon+\delta$

$\leq 1+|\bar{\Pi}(\vec{b}, \vec{c})+\vec{b} \cdot \vec{c}|-\vec{b} \cdot \vec{c}+\varepsilon+\delta$

$\leq 1-\vec{b} \cdot \vec{c}+2(\varepsilon+\delta)$, 
and the left hand side is also,

$$
\begin{aligned}
& |\bar{\Pi}(\vec{a}, \vec{b})-\bar{\Pi}(\vec{a}, \vec{c})|=|\bar{\Pi}(\vec{a}, \vec{b})+\vec{a} \cdot \vec{b}-\vec{a} \cdot \vec{b}-\bar{\Pi}(\vec{a}, \vec{c})+\vec{a} \cdot \vec{c}-\vec{a} \cdot \vec{c}| \\
& \geq|\vec{a} \cdot \vec{c}-\vec{a} \cdot \vec{b}|-|\bar{\Pi}(\vec{a}, \vec{b})+\vec{a} \cdot \vec{b}|-|\bar{\Pi}(\vec{a}, \vec{c})+\vec{a} \cdot \vec{c}| \\
& \geq|\vec{a} \cdot \vec{c}-\vec{a} \cdot \vec{b}|-2(\varepsilon+\delta),
\end{aligned}
$$

The violation of the Bell's inequality implies that, quantum mechanics is a totally non-classical phenomenon:- non-realistic and non-local, (Shankar, 2012) [22].

To obtain Bell's inequality and consequently conflicts with quantum mechanics, the hypothesis below seem necessary.

- distant correlations can be understood by introduction of supplementary parameters carried along by the separated particles, in the spirit of Einstein's ideas that separated objects have separated physical realities.

- the quantities $A(\lambda), B(\lambda)$ and $P(\lambda)$ obey the locality condition, that is, they do not depend on the orientations of the distant polarizers.

This is why we say quantum mechanics conflicts with local realism, (Dada et al., 2011) [17].

\section{Quantum teleportation}

Quantum teleportation is a form of quantum information using features of quantum physics to deal with computational problem. Quantum teleportation was first proposed (Bennett et al., 1993) [5]. A device scans an object, de-materialize it and transmitted to another location where the object is re-materialized back to its original state.

In 1997, two groups of physicist Anton Zerlinger and A. Francisco De Martini both carried out the first successful teleportation of a single photon called quantum bit teleportation in probabilistic sense. Each group used the technique of a report in 1993 by Bennett of IBM and it was related on quantum entanglement. Quantum entanglement is a strange intimate relationship between two particles and each of these two particles being in the same quantum state. When one of the particles change its spin, the other will fall exactly on the same way instantaneously regardless of spatial separation. The teleportation used by Zerlinger and De Martini is known as quantum teleportation. Quantum teleportation is where the specific quantum state of the photon is measured and sent to another location where that information can be used to manipulate another photon and into the same quantum state. However, the act of measuring the quantum of a specific photon will cause that photon to move into a new quantum state. Therefore the quantum state of the second photon will not reflect the quantum state of the first photon before measurement. Suppose we want to teleport a photon $A$ to a distant location. The first step is to have the entangled pair photon $B$ and $C, C$ then at the destination. We do not measure directly photon $A$ because in doing so, we will disrupt its quantum state, instead we measure a joint feature between photon $A$ and the entangled photon $B$. In doing so, we see the relation between photon $A$ and photon $B$. Since photons $B$ and $C$ are entangled, we get to know how photon $A$ relates to photon $C$. This information is then communicated to the desired location through traditional means such as telephone, email and fax. Once the other side receives this information, we can use it to manipulate photon $C$ into the same quantum state as photon $A$. Since the act of measuring photon $A$ disrupts its quantum state; quantum teleportation has been achieved. Although marine science has successfully teleported a single photon, even smallest objects are made of billions of particles. Therefore telepotation by this method is not practical but theoretically possible.

Quantum teleportation can be thought of as a quantum gate where input and output are the same.

Classical teleportation is easy by making a model of the object and measuring all the dimensions and positions of the component. This information can be sent digitally from $A$ to $B$ using a prototype of that object. But in Quantum states, there are physical rules which does not allow copying. The superposition states of a $\alpha|1\rangle$ and $\beta|0\rangle$ cannot be cloned. We need to choose a measurement basis in which to measure it and project into $\alpha|1\rangle$ or $\beta|0\rangle$.

“... the quantum state of a photon can be maintained whiles transporting it into a crystal without the two coming directly into contact".

He explains that "one needs to imagine two entangled photons in other words two photons extricable linked at the most infinitesimal level by their joint states. One is propelled along an optical fibre ... but not the other, which is sent to a crystal. It is a bit like a game of billiards, with a third photon hitting the first which obliterates both of them ..."
He conclude that "the quantum state of the two elements of light, these two entangled photons which are like two siamese twins, is a channel that empowers the teleportation from light into matter."

Quantum entanglement has become essential in quantum information and also important in the applications of quantum telepotation and quantum cryptography (Barrett et al., 2004) [2]. Our ability to manipulate these entangled states is the basis for the applications. Experiments such as photons pair produced in an optical process as atoms in cavity QED, parametric downconversion and with ions in an ion trap are due to the manipulation of these entangled state using two or more spatially separated particles.

"The experimental violation of Bell's inequalities confirms that a pair of entangled photons separated by hundreds of metres must be considered a single non-separable object - it is impossible to assign local physical reality to each photon."

In this section, we discuss the method by which a teleporting system can be created using the Bell's inequality. We argue that the violation of the Bell's inequality of any two mixed spin- $\frac{1}{2}$ states is necessary for teleportation Teleportation is purely based on classical information and non-classical EPR correlations.

The basic idea is to use a pair of particles in a singlet state shared by distant partners Alice and Bob to perform successful teleportation of an arbitrary qubit from the sender Alice to the receiver Bob. There was a question what value of fidelity of transmission of an unknown state can ensure us about non-classical character of the state forming the quantum channel. It has been shown that the purely classical channel can give at most $F=\frac{2}{3}[10,3,5]$. Then Popescu raised basic questions concerning a possible relation between teleportation, Bell-CHSH inequalities and inseparability.

\subsection{Quantum entanglement}

Quantum entanglement is a pure quantum phenomenon that occurs when pairs or groups of particles are generated in such a way that the quantum state of each particle cannot be described independently. Entangled state has no classical analogue, for it clearly distinguishes quantum mechanics from the classical.

The existence of entanglement is based on superposition principle and an entangled state. In superposition principle, outcomes of measurements are modelled by vector basis in a complex vector space and the state can be in superposition. Two state systems can be combined by using a tensor product and the Hilbert space for each system are tensored as $\mathscr{H}_{1} \otimes \mathscr{H}_{2}$. An entangled state of two qubit state is defined as

$\left|\Psi^{\dagger}\right\rangle_{A B}=\frac{1}{\sqrt{2}}\left(|00\rangle_{A B}+|11\rangle_{A B}\right)$.

\subsection{Maximal fidelity for the standard teleportation scheme}

Fidelity is a measure of the "closeness" of two quantum states. The fidelity of $X$ and $Y$ is defined as the quantity

$F(X, Y)=\sum_{i} \sqrt{p_{i} q_{i}}$

where $X$ and $Y$ are two random variables with values $(1 \cdots n)$ and probabilities $p=\left(p_{1} \cdots p_{n}\right)$ and $q=\left(q_{1} \cdots q_{n}\right)$.

For $F \in[0,1]$, with $F=1$ meaning that two states are identical, and $F=0$ means that, they are different. For example, create pairs of perfectly entangled particles and having an extra stray (non-entangled) photons that you measure in your ensemble of prepared bi-photons, then your ensemble averaged state will be different than what you want (perfectly entangled bi-photon pairs) and how close you are is given by fidelity $(\mathrm{F})$,

$F(\rho, \sigma):=[\operatorname{Tr}|\sqrt{\rho} \sqrt{\sigma}|]^{2}$

$=(\operatorname{Tr} \sqrt{\sqrt{\rho} \sigma \sqrt{\rho}})^{2}$

where $\rho$ and $\sigma$ represent a density matrix.

Teleportation can be expressed as a quantum qubit $|\Psi\rangle$ in Alice's possession which wants to convey to Bob as

$|\Psi\rangle_{C}=A|0\rangle_{C}+B|1\rangle_{C}$

$C$ distinguishes the state from $A$ to $B$. In standard teleportation, Alice uses operator basis in her frame of measurement whiles Bob is allowed to transform 
from one basis to another. The representation of this state in the HilbertSchmidt space with a scalar product $\langle A, B\rangle=\operatorname{Tr}\left(A^{\dagger} B\right)$ is represented as

$\rho=\frac{1}{4}\left[I \otimes I+\mathbf{r} \cdot \boldsymbol{\sigma} \otimes I+I \otimes \mathbf{s}-\boldsymbol{\sigma}+\sum_{n, m}^{3} t_{n m} \sigma_{n} \otimes \sigma_{m}\right]$,

$\rho$ acts on the Hilbert space $\mathscr{H}_{1} \otimes \mathscr{H}_{2}=C^{2} \otimes C^{2}, I$ represents the identity operator, $\left\{\sigma_{n}\right\}_{n=1}^{3}$ represents the Pauli matrices, $\mathbf{r}$ and $\mathbf{s}$ are vectors in $R^{3}, \mathbf{r}$. $\boldsymbol{\sigma}=\sum_{i=3}^{3} \sigma_{i} . t_{m n}=\operatorname{Tr}\left(\rho \sigma_{n} \otimes \sigma_{m}\right)$ from a real matrix denoting as $T$. $\mathbf{r}$ and $\mathbf{s}$ describes the local behaviour of the state and the mean value of the Bell-CHSH observables only depends on the relationship parameter $T$ of the state.

$$
\begin{gathered}
\rho_{1} \equiv \operatorname{Tr}_{\mathscr{H}_{2}} \rho=\frac{1}{2}(I+\mathbf{r} \cdot \boldsymbol{\sigma}), \\
\rho_{2} \equiv \operatorname{Tr}_{\mathscr{H}_{1}} \rho=\frac{1}{2}(I+\mathbf{s} \cdot \boldsymbol{\sigma})
\end{gathered}
$$

The matrix $T$ is responsible for the expectation of the correlation

$$
E(a, b) \equiv \operatorname{Tr}(\rho \mathbf{a} \cdot \boldsymbol{\sigma} \otimes \mathbf{b} \cdot \boldsymbol{\sigma})=(\mathbf{a}, T \mathbf{b}) .
$$

Considering two particle source producing pairs in a given state $\rho$ given to Bob whiles the other one and another third particle in an unknown state $\phi$ subjected to Alice's measurement can be expressed as a family of projectors

$P_{k}=\left|\Psi_{k}\right\rangle\left\langle\Psi_{k}\right|$,

where $k=0,1,2,3$ and $\Psi_{k}$ represents the Bell basis.

$$
\begin{aligned}
& \Psi_{(2)}^{1}=\frac{1}{\sqrt{2}}\left(e_{1} \otimes e_{2} \mp e_{2} \otimes e_{1}\right) \\
& \Psi_{(0)}^{3}=\frac{1}{\sqrt{2}}\left(e_{1} \otimes e_{2} \pm e_{2} \otimes e_{1}\right)
\end{aligned}
$$

taking $e_{1}, e_{2}$ to be the standard basis in $C^{2}$. Alice then sends to Bob the number of outcome $k$ from the two bits and Bob applies a unitary transformation $U_{k}$ for his particle to be in state $\rho_{k}$.

The fidelity as a measure of efficiency of teleportation of a transmission of the unknown state is expressed as

$$
\mathscr{F}=\int_{s} d M(\phi) \sum_{k} p_{k} \operatorname{Tr}\left(\rho_{k} P_{\phi}\right) \text {. }
$$

$P_{\phi}$ is the input state, $\rho_{k}$ is the output state, provided the outcome $k$ was obtained by Alice. Where the integral is taken over all $\phi$ belonging to the Bloch sphere with uniform distribution $M, P_{k}=\operatorname{Tr}\left[\left(P_{k} \otimes I\right)\left(P_{\phi} \otimes \rho\right)\right]$ denoting the probability of the $k$-th outcome. In computing for the integral (25), the output state $\rho_{k}$ is expressed as

$\rho_{k}=\frac{1}{p_{k}} \operatorname{Tr}_{1,2}\left[\left(P_{k} \otimes U_{k}\right)\left(P_{\phi} \otimes \rho\right)\left(P_{k} \otimes U_{k}^{\dagger}\right)\right]$,

$\rho_{k} p_{k}=\operatorname{Tr}_{1,2}\left[P_{k} P_{\phi} P_{k} \otimes U_{k} \rho U_{k}^{\dagger}\right]$.

Since $P_{k}$ and $\rho$ are tensor products, let $P_{k}=A \otimes B$ and $\rho=C \otimes D$ and substitute into equation (26). This implies that

$\rho_{k} p_{k}=\operatorname{Tr}_{1,2}\left[A P_{\phi} A \otimes B C B \otimes U_{k} D U_{k}^{\dagger}\right]$.

If

$\rho=C \otimes D=\frac{1}{4}\left[I \otimes I+\mathbf{r} \cdot \boldsymbol{\sigma} \otimes I+I \otimes \mathbf{s} \cdot \boldsymbol{\sigma}+\sum_{n, m=1}^{3} t_{n m} \sigma_{n} \otimes \sigma_{m}\right]$,

then

$\rho_{k} p_{k}=\frac{1}{4} \operatorname{Tr}_{1,2}\left[A P_{\phi} A \otimes B(I+\mathbf{r} \cdot \boldsymbol{\sigma}+I]+\right.$

$\left.+\frac{1}{4} \operatorname{Tr}_{1,2}\left[\sum_{n, m=1}^{3} t_{n, m} \sigma_{n}\right) B \otimes U_{k}\left(I+I+\mathbf{s} \cdot \boldsymbol{\sigma}+\sigma_{m}\right) U_{k}^{\dagger}\right]$.

This also implies that

$$
\begin{aligned}
\rho_{k} p_{k} & =\frac{1}{4} \operatorname{Tr}_{1,2}\left[A P_{\phi} A \otimes B(2 I+\mathbf{r} \cdot \boldsymbol{\sigma}+]\right. \\
& \left.+\frac{1}{4} \operatorname{Tr}_{1,2}\left[\sum_{n, m=1}^{3} t_{n m} \sigma_{n}\right) B \otimes\left(U_{k} 2 I U_{k}^{\dagger}+U_{k} \mathbf{s} \cdot \boldsymbol{\sigma} U_{k}^{\dagger}+U_{k} \sigma_{m} U_{k}^{\dagger}\right)\right]
\end{aligned}
$$

Substituting $P_{\phi}=\frac{1}{2}(I+\mathbf{a} \cdot \boldsymbol{\sigma})$ into equation (27) and rewritten in terms of $P_{k}$ will result in

$\rho_{k} p_{k}=\frac{1}{8} \operatorname{Tr}\left[P_{k}^{2}\left[(I+\mathbf{a} \cdot \boldsymbol{\sigma}) \otimes 2 I+\mathbf{r} \cdot \boldsymbol{\sigma}+\sigma_{n}\right]\right] U_{k}\left(2 I+\mathbf{s} \cdot \boldsymbol{\sigma}+\sum t_{n m} \sigma_{m}\right) U_{k}^{\dagger}$ $\rho_{k} p_{k}=\frac{1}{8} \operatorname{Tr}\left[2 P_{k}^{2}(I \otimes I)+P_{k}^{2}(I \otimes \mathbf{r} \cdot \boldsymbol{\sigma})+P_{k}^{2} I \otimes \sigma_{n}+P_{k}^{2}(\mathbf{a} \cdot \boldsymbol{\sigma} \otimes I)\right.$

$\left.+P_{k}^{2}(\mathbf{a} \cdot \boldsymbol{\sigma} \otimes \mathbf{r} \cdot \boldsymbol{\sigma})+P_{k}^{2}(\mathbf{a} \cdot \boldsymbol{\sigma}) \otimes \sigma_{n}\right]$.

Therefore

$p_{k} \rho_{k}=\frac{1}{8}\left(\left[1+\left(\mathbf{a}, T_{k} \mathbf{r}\right)\right] I+O_{k}^{\dagger}\left[\mathbf{s}+T^{\dagger} T_{k} \mathbf{a}\right] \cdot \sigma\right)$.

The $T_{k}^{\prime} s$ and $\mathbf{r}, \mathbf{s}, T$ corresponds to $P_{k}^{\prime} s$ and $\rho$ respectively through (20) (Thus: $T_{0}=\operatorname{diag}(-1,-1,-1), T_{1}=\operatorname{diag}(-1,1,1), T_{2}=\operatorname{diag}(1,-1,1), T_{3}=$ $\operatorname{diag}(1,1,-1), \mathbf{r}_{k}=\mathbf{s}_{k}=0$, for $k=0,1,2,3$.); $O_{k}$ 's are rotations in $R^{3}$ which we get from $U_{k}$ s $s$ by

$U \hat{\mathbf{n}} \cdot \boldsymbol{\sigma} U^{\dagger}=\left(O^{\dagger} \hat{\mathbf{n}}\right) \cdot \boldsymbol{\sigma}$.

Considering the formula

$\int_{S}(\mathbf{a}, A \mathbf{a}) d M(\mathbf{a})=\frac{1}{3} \operatorname{Tr} A$

and omitting the all terms in (25) which do not contribute to the integral, will result in

$\mathscr{F}=\frac{1}{8} \sum_{k}\left(1+\frac{1}{3} \operatorname{Tr} T_{k}^{\dagger} T O_{k}\right)$.

Now we maximize $\mathscr{F}$, and since $-T_{k}^{\dagger}$ is a rotation and the maxima of the term in (32) does not depend on $k$, we then have

$\mathscr{F}_{\text {max }}=\max _{0} \frac{1}{2}\left(1-\frac{1}{3} \operatorname{Tr} T O\right)$

where we take the maxima over all the rotations. This gives us

$\mathscr{F}_{\text {max }} \leq \frac{1}{2}\left(1+\frac{1}{3} \operatorname{Tr} \sqrt{T^{\dagger} T}\right)$.

Without using entanglement, by purely classical communication, an average fidelity of $F=\frac{1}{2}$ is the best that can be achieved if the alphabet of input states includes all coherent states with even weight. If the latter equation (34) is greater than $\frac{2}{3}$ which is the upper bound for classical teleportation, then we can derive an expression for $\mathscr{F}_{\text {max }}$ and the one say that the state of the quantum channel is necessary for teleportation. $\mathscr{F}_{\max }$ can exceed $\frac{2}{3}$ only if $\operatorname{Tr} \sqrt{T^{\dagger} T}$. From the result obtained in Ref. The equation (34) implies that $\operatorname{det} T<0$ and the inequality (34) then progresses into equality. As result, if we define a function

$N(\rho):=\operatorname{Tr} \sqrt{T^{\dagger} T}$,

we have

Proposition 1. Any mixed spin- $\frac{1}{2}$ state is necessary for teleportation if and only if $N(\rho)>1$. The fidelity will then be expressed as

$\mathscr{F}_{\text {max }}=\frac{1}{2}\left(1+\frac{1}{3} N(\rho)\right)$.

\subsection{Relation between Bell's inequalities and teleporta- tion}

To establish a strong relationship between the Bell-CHSH inequality and quantum teleportation, the function $N(\rho)$ must be a correlation parameter of $T$. Violating the Bell-CHSH which involves a real valued function (which is a necessary condition) implies that, $M(\rho)=\max _{i>j}\left(u_{i}+u_{j}\right)$, where $u_{i}$ and $u_{j}$ represents the eigenvalues of the matrix $T^{\dagger} T$. This then becomes equivalent to the Bell-CHSH when the inequality $M(\rho) \leqslant 1$. If $u_{i} \leqslant 1$ for $i=1,2,3$ and $N(\rho)=\sum_{i=1}^{3} \sqrt{u_{i}}$ then we have

$N(\rho) \geqslant M(\rho)$.

$M(\rho)>1$ is the state at which we violate the Bell-CHSH inequality. From (37) and Prop.5.1.1 we get

$\mathscr{F}_{\text {max }} \geqslant \frac{1}{2}\left(1+\frac{1}{3} M(\rho)\right)>\frac{2}{3}$.

The CHSH-Bell observable at maximal will have a mean value of $B_{\max }=$ $2 \sqrt{M(\rho)}$, and this implies that

$\mathscr{F} \geqslant \frac{1}{2}\left(1+\frac{1}{12} B_{\max }^{2}\right)$.

The inequalities (38) and (39) are useful for teleportation since they are valid for any arbitrary mixed two spin- $\frac{1}{2}$ which violate the Bell-CHSH inequality. 
The Bell-CHSH inequalities constructed from the expectations of products of spin operators $\mathbf{a} \cdot \sigma \otimes \mathbf{b} \cdot \sigma$ where $\mathbf{a}$ and $\mathbf{b}$ are unit vectors [?]. The correlation function can be expressed as

$E(\mathbf{a}, \mathbf{b}) \equiv \operatorname{Tr}(\rho \mathbf{a} \cdot \boldsymbol{\sigma} \otimes \mathbf{b} \cdot \sigma)=(\mathbf{a}, T \mathbf{b})$

and it depends on the $T$ matrix only. Hence the Bell-CHSH can be violated if $N(\rho)>1$. If $N(\rho) \leqslant 1$, it means there always exist some separable state that has the same $T$ matrix as the state $\rho$ (R. Horodecki et al., 1996).

Proposition 2. Every mixed two spin- $\frac{1}{2}$ state which violates any Bell-CHSH inequality is useful for teleportation (R. Horodecki et al., 1996).

\section{Applications of teleportation and further work}

Quantum teleportation is applied cryptology. It provides a completely secure method of communication between two distant correspondents. Sending photons entangled in a quantum state makes it impossible for an eavesdropper to intercept a message. Future work will be on teleportation of more complicated quantum systems such as molecules, DNA and organic molecules and the development of other real-life applications.

\section{Conclusion}

In this paper, we considered questions concerning the completeness of quantum mechanics by exploring the consequences of quantum mechanics different from classical mechanics due to superposition of states and the process of measurement. From the study of Bell's inequality, he concluded that "without changing the statistical predictions, there must be a mechanism whereby the setting of one measuring device can influence the reading of another instrument, however remote". Quantum mechanics is therefore considered non-local by the violation of Bell's inequality. Therefore, an entangled EPR photon pair is a non-separable object; that is, it is impossible to assign individual local properties to each photon. We reviewed how the violation of Bell's inequality can be used for quantum teleportation.

\section{Acknowledgement}

Appreciation goes the Almighty God. My sincere thanks to my co-authors who assisted in putting this together and all who helped in diverse ways.

\section{References}

[1] Alain Aspect, "Bell's theorem: the naive view of an experimentalist", Springer, (2002).

[2] Barrett, M. D., Chiaverini, J., Schaetz, T., \& Britton, J., "Deterministic quantum teleportation of atomic qubits", Nature, Vol.429, No.6993, (2004).

[3] Bell, J. S., "Speakable and unspeakable in quantum mechanics: Collected papers on quantum philosophy", Cambridge university press, (2004).

[4] Bell, J. S., "On the impossible pilot wave", Foundations of Physics, Vol.12, No.10, (1982), pp.989-999

[5] Bennett, C. H., Brassard, G., Crépeau, C., Jozsa, R., Peres, A. and Wootters, W. K., "Teleporting an unknown quantum state via dual classical and Einstein-Podolsky-Rosen channels", Physical review letters, Vol.70, No.13, (1993), p.1895.

[6] Blaylock, Guy. "The EPR paradox, Bell's inequality, and the question of locality", American Journal of Physics, Vol.78, No.1, (2010), p:111120.

[7] Dada, A. C., Leach, J., Buller, G. S., Padgett, M. J., \& Andersson, E., "Experimental high-dimensional two-photon entanglement and violations of generalized Bell inequalities", Nature Physics, Vol.7, No.9, (2011), 677-680.

[8] Dirac, P. A. M., "The principles of quantum mechanics ", Oxford university press, No.27, (1981).

[9] Einstein, A., Podolsky, B. and Rosen, N., "Can quantum-mechanical description of physical reality be considered complete?", Physical review, Vol.47, No.10, (1935), p.777.

[10] Genovese, M., "Research on hidden variable theories: A review of recent progresses", Physics Reports, Vol.413, No.6, (2005), pp.319396.

[11] Griffiths, D. J., "Introduction to quantum mechanics", Pearson Education India.

[12] Hasegawa, Yuji, Rudolf Loidl, Gerald Badurek, Matthias Baron, and Helmut Rauch, "Violation of a Bell-like inequality in single-neutron interferometry", Nature 425, No. 6953, (2003), pp:45-48.

[13] Home, D. and Selleri, F., "Bell's theorem and the EPR paradox", La Rivista del Nuovo Cimento, Vol.14, No.9, (1978-1999), pp.1-95.
[14] Horodecki, R., Horodecki, M. and Horodecki, P., "Teleportation, Bell's inequalities and inseparability", Physics Letters A, Vol.222, No.1-2, (1996), pp.21-25.

[15] Hu, M. L., "Teleportation of the one-qubit state in decoherence environments", Journal of Physics B: Atomic, Molecular and Optical Physics, Vol.44, No.2, (2011), p.025502.

[16] J. S. Bell et al., "On the einstein-podolsky-rosen paradox", Physics, Vol.1, No.3, (1964), pp:195-200.

[17] Klöck, D., "Quantum Computers".

[18] Lee, J. and Kim, M. S., "Entanglement teleportation via Werner states", Physical review letters, Vol.84, No.18, (2000), p.4236.

[19] R. Horodecki, M. Horodecki, and P. Horodecki, "Teleportation, bell's inequalities and inseparability", Physics Letters A, Vol.222, No.1, (1996), pp:21-25.

[20] Reilly, Michael H. "Temperature dependence of the short wavelength transmittance limit of vacuum ultraviolet window materials-II theo-
retical, including interpretations for UV spectra of $\mathrm{SiO} 2, \mathrm{GeO} 2$, and A12O3", Journal of Physics and Chemistry of Solids 31, No. 5, (1970), pp: 1041-1056.

[21] Riebe, M., Haffner, H., Roos, C. F., \& Hansel, W., "Deterministic quantum teleportation with atoms", Nature, Vol.429, No.6993, (2002), p.734.

[22] Shankar, R., "Principles of quantum mechanics", Springer Science \& Business Media, (2012).

[23] S. Turgut., "Measurement process in quantum mechanics", Vol.1, (2013).

[24] Tang, C. L., "Fundamentals of quantum mechanics: for solid state electronics and optics", Cambridge University Press, (2005).

[25] Terhal, B. M., Doherty, A. C., \& Schwab, D., "Symmetric extensions of quantum states and local hidden variable theories", Physical review letters, Vol.90, No.15, (2003).

[26] Wakita, H., "Measurement in quantum mechanics", Progress of Theoretical Physics, Vol.23, No.1, (1960), pp.32-40. 